\title{
GLOBAL ENERGY MATCHING METHOD FOR ATOMISTIC-TO-CONTINUUM MODELING OF SELF-ASSEMBLING BIOPOLYMER AGGREGATES*
}

\author{
LEI ZHANG ${ }^{\dagger}$, LEONID BERLYAND ${ }^{\ddagger}$, MAXIM V. FEDOROV ${ }^{\S}$, AND HOUMAN OWHADI
}

\begin{abstract}
This paper studies mathematical models of biopolymer supramolecular aggregates that are formed by the self-assembly of single monomers. We develop a new multiscale numerical approach to model the structural properties of such aggregates. This theoretical approach establishes micro-macro relations between the geometrical and mechanical properties of the monomers and supramolecular aggregates. Most atomistic-to-continuum methods are constrained by a crystalline order or a periodic setting and therefore cannot be directly applied to modeling of soft matter. By contrast, the energy matching method developed in this paper does not require crystalline order and, therefore, can be applied to general microstructures with strongly variable spatial correlations. In this paper we use this method to compute the shape and the bending stiffness of their supramolecular aggregates from known chiral and amphiphilic properties of the short chain peptide monomers. Numerical implementation of our approach demonstrates consistency with results obtained by molecular dynamics simulations.
\end{abstract}

Key words. atomistic-to-continuum, self-assembly, biopolymer aggregates, multiscale

AMS subject classifications. 75L15, 74Q05, 65Z05

DOI. $10.1137 / 090781619$

1. Introduction. Fast development of bio- and nanosciences requires new methods for theoretical investigations of structural properties of supramolecular aggregates consisting of thousands to millions of atoms. However, the fully atomistic treatment of such big aggregates remains a challenge. These days, the most promising strategy for computer modeling of nano-sized supramolecular aggregates is the multiscale approach, e.g., [29, 39]. The fundamental idea behind the multiscale approach is that detailed information from the atomistic scale is systematically coarsegrained and then used for continuous modeling of the aggregates in a self-consistent way.

Using the multiscale approach, we have developed a new low-cost model for simulating the mechanical properties of supramolecular tapes formed by the self-assembling oligopeptides (a short chain of peptide) $[1,2,4,3]$. These oligopeptides can form regular tapelike and rodlike nanoaggregates in aqueous solutions $[1,2,4,3]$. The aggregates are important as promising "smart" biodegradable nanomaterials [52] because one could easily assemble and disassemble them by varying the solvent conditions $(\mathrm{pH}$, temperature, etc.) $[4,3]$. These self-assembled systems are also important as model

\footnotetext{
* Received by the editors January 4, 2010; accepted for publication (in revised form) September 2, 2010; published electronically December 7, 2010.

http://www.siam.org/journals/mms/8-5/78161.html

${ }^{\dagger}$ Hausdorff Center for Mathematics, University of Bonn, Endenicher Allee 60, Bonn, 53115, Germany (lei.zhang@hcm.uni-bonn.de).

${ }^{\ddagger}$ Pennsylvania State University, Department of Mathematics, University Park, PA 16802 (berlyand@math.psu.edu). The work of this author was partially supported by NSF grant DMS0708324 and DOE grant DEFG02-08ER25862.

$\S$ Max-Planck-Institute for Mathematics in the Sciences, Inselstr. 22, 04103, Leipzig, Germany (fedorov@mis.mpg.de).

『 California Institute of Technology, Department of Computing and Mathematical Sciences, Pasadena, CA 91125 (owhadi@acm.caltech.edu). The work of this author was partially supported by NSF grant CMMI-0926001.
}

1958 
systems for amyloid-like fibril assemblies formed by self-assembling natural oligopeptides and misfolded proteins [32]. The amyloid fibrils are the potential cause of such diseases as Huntington's and Alzheimer's and type II diabetes [45].

Because these supramolecular assemblies are very difficult to crystallize, there are severe difficulties with experimental studies of their structural and mechanical properties. Therefore, one should complement the experimental studies with simulations to obtain complete information about the structural and mechanical properties of the supramolecular systems. Although there were several attempts to simulate these systems using the standard "one-scale" tools of molecular simulations [37, 26, 10, 11, 12], in general, these assemblies are too large for standard methods of atomistic simulations. For example, to make a clear connection between available experiments and simulations, one should model many systems comprised of $10^{5}-10^{7}$ atoms by varying the solvent conditions as well as the chemical structure of the monomers. This problem is practically intractable for the standard one-scale methods of atomistic simulations. To overcome these obstacles, we have developed a new multiscale model which hybridizes, in an efficient way, the atomistic molecular dynamics (MD) method with continuum mechanics.

Previous work has been done, e.g., in [7, 27], to derive continuum models for crystalline atomistic tapes and rods which resemble the standard Cauchy-Born rule for bulk solids. The resulting elastic model is usually strongly nonlinear and depends on the crystalline order, which makes it difficult to implement numerically. In this work, we use the energy matching principle to derive an appropriate curvature elasticity model numerically from the atomistic model.

The main steps of this method are as follows: First, a continuum model with $m$ unknown parameters is discretized on a piecewise flat triangulated surface which approximates the atomistic tape. $m$ is a number related to the nature of continuum model and the dimension of the space (for instance $m=6$ for linear elasticity of bulk body in dimension three). Next, $m$ representative and independent displacements (global solutions) of the atomistic model are computed with appropriate boundary conditions. Finally, the $m$ unknown parameters of the continuum model are determined by enforcing that, for each one of these $m$ precomputed (global) solutions, the elastic energy of the continuum model matches the total energy of the atomistic model.

Mathematically, our method is based on the observation that, at fine scales, arbitrary solutions of the atomistic system can be locally approximated by a linear combination of a few independent precomputed fine scale solutions $[41,8,34,16]$. We use the method to study the geometric chirality and mechanic property of the self-assembled atomistic tape. The proposed model is much less computationally demanding than the fully atomistic methods and can be used to investigate the properties of large self-assembled structures.

The paper is organized as follows: First, we introduce the reader to the necessary mathematical and biological background of the problem. Second, we describe the problem, atomistic and continuum modeling, in section 3, "Atomistic and Continuum Modeling of Atomistic Tape." Then we introduce the energy matching method in section 4, "Atomistic-to-Continuum (AtC) Modeling by Energy Matching Method." In the "Numerical Results" section (5), we will use the proposed method to study the structure transition and mechanical properties of the self-assembled tapes with different atomistic parameters and show that the method works well for continuum models of different complexity. 


\section{Biological and mathematical background.}

2.1. Biological background: Self-assembly of biopolymers. We model selfassembled aggregates of biomolecules, e.g., peptides, with the main focus on the oligopeptide self-assembled systems, which may serve as a model system to study the aggregation process in amyloid-caused disease because there are experiments that reveal substantial structural similarity between the fibrils formed by the peptide self-assembly and the amyloid fibrils found in conformational diseases, such as Huntington's, Alzheimer's, and type II diabetes [45]. These synthetic oligopeptides are specifically designed to make them self-assemble [51]. They can be considered as short segments of polypeptide chains that are formed by a predefined sequence of amino acids linked head-to-tail with covalent peptide bonds.

In short, the self-assembly mechanism is $[1,2,3,4,32]$ the following: Peptide is a sequence of amino acids linked by peptide bonds. A peptide bond is a covalent bond that is formed between two adjacent amino acids when the carboxyl group of one amino acid reacts with the amino group of another amino acid. Single peptides in solution can undergo a conformational change from a random coil (different coils can vary randomly in shape) and assemble side by side to form a $\beta$-tape. $\beta$-tape [5] is a wide class of macromolecular structures, formed by aligning oligopeptide strands in parallel. This is the first level of the hierarchy; see Figure 1. With increasing concentration, more complex amyloid-like structures such as ribbons (double tapes), double ribbons, fibrils, and fibers can be developed. Noncovalent bonds are crucial for the self-assembly process. In this work, we will only study the self-assembled $\beta$-tapes. The future goal is to study the more complicated hierarchical structure of the self-assembled system.

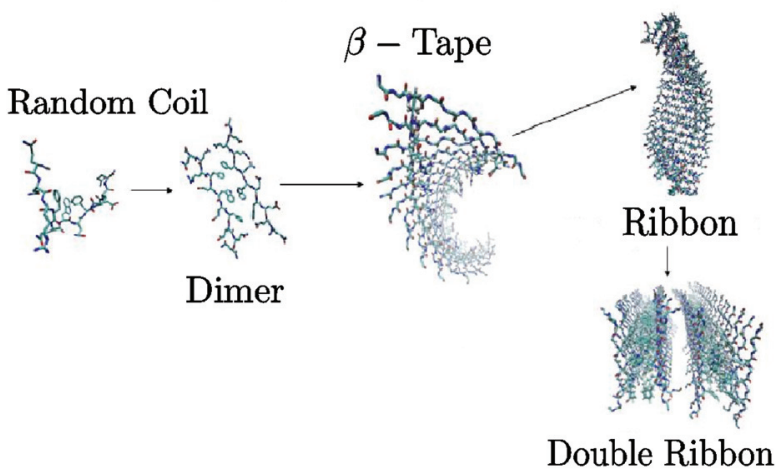

FIG. 1. Hierarchical structures of self-assembly.

Atoms can be modeled as spheres with the radius defined as van der Waals radius. The fact that each atom within a molecule occupies a certain amount of space, which is associated to the energy cost due to overlapping electron clouds (Pauli or Born repulsion), may affect the molecule's preferred shape (conformation) and reactivity. Furthermore, this constraint limits greatly the possible bond angles in a polypeptide chain. This constraint and other steric interactions (excluding volume effect) severely restrict the variety of three-dimensional possible arrangements of atoms (or conformations). In the following, we will see how to mathematically model these bonded interactions on the atomistic scale. 
The conformation of biopolymers is, however, further constrained by many different sets of weak noncovalent bonds (or nonbonded interactions) that form between atoms, which are not linked by covalent bonds, in different peptide chains or in different parts of a single peptide chain. The weak bonds are mainly of four types: hydrogen bonds, ionic bonds, van der Waals attractions, and hydrophobic/hydrophilic interaction. Individual noncovalent bonds are 30-300 times weaker than the typical covalent bonds that create biological molecules [5]. Many weak bonds can act in parallel to hold two peptide chain or two regions of a peptide chain tightly together. The stability of each folded shape is therefore determined by the combined strength of large numbers of such noncovalent bonds.

2.2. Mathematical background: Atomistic and continuum modeling for atomistic tapes. Molecular self-assembly is one class of physical problems with many scales. Such multiscale problems are ubiquitous in material sciences. To make them more amenable to analysis, it is often necessary to make the assumption of scale separation. Namely, choose a small parameter $\varepsilon$ as the ratio between the microscopic scale and macroscopic scale, let $\varepsilon \rightarrow 0$, and study the $\varepsilon$ family of problems with translational invariance (periodicity, quasi periodicity, or ergodicity), such as in classical homogenization theory $[15,33,42,6,23]$. For example, the ratio between the interatom distance and the object size can be taken as such a small parameter. Recently, these analytical approaches $(\Gamma-, G$ - and $H$-convergence $[15,33,42,6,23])$ have been applied to study the atomistic-to-continuum limits for thin elastic films [27, 44].

In real applications, we often need to compute the solution numerically. Furthermore, in most problems of material sciences, physics, and biology, one has to deal with a given medium and not with an $\varepsilon$ family of media. A direct atomistic simulation of the multiscale problem, which typically involves a wide range of spatial and time scales, is still difficult even with state-of-the-art supercomputers and algorithms. We often need to use multiscale methods to solve the problem on a coarser scale. More precisely, we need to know how to extract and transfer information from fine scales to coarse scales and how to use the obtained information to construct a coarse scale solver that is efficiently computable.

A system such as molecular self-assembly can be most naturally studied by atomistic models, given the full atomistic potential. However, the computational expense limits atomistic simulations to the scale of nanoseconds in duration and nanometers in length; therefore, it is important to introduce a coarse-grained continuum model to interpret and extend the results of atomistic simulation.

Oligopeptides can form a hierarchy of structures via self-assembly, and in this hierarchy the $\beta$-tape is the first level. The intrinsic chirality of the $\beta$-tape is directly related to the atomistic parameters of the peptide, namely, the asymmetry of the side chains and the twist of the peptide backbone. On the other hand, the chirality of the $\beta$-tape determines the structure and formation of higher order structures. Therefore, our first goal is to study self-assembled $\beta$-tapes and derive their elastic energy, which can be used afterward as packing energy (building block energy) for higher order structures, e.g., energy of a single tape which determines the formation of the next order structure such as ribbon.

At the continuum scale, the tapelike structures can be modeled as elastic strips with small or zero thickness which can be treated by elastic plate, shell, or membrane theory depending on the specific situation. Analogous to the Saint Venant-Kirchhoff model of finite elasticity, elastic energy for a two-dimensional continuum can be split into membrane energy and bending energy. On the nanoscale, the supramolecular 
structure is hardly extendible, which is why we are primarily interested in twisting and bending energies. It can be verified by atomistic simulation [12] that fluctuations in the interpeptide distance and in the single peptide end-end distance are negligible compared to the fluctuations related to twisting and bending [13, 14]. Therefore, we make an assumption that the atomistic tape is inextensible, and the in-plane contribution to the elastic potential energy is negligible.

The problem of deriving continuum description from atomistic potential has been studied by many authors. Friesecke and James [27] introduced a scheme to construct continuum models directly from atomistic models for multilayer thin films and nanorods. Arroyo and Belyschko [7] proposed the exponential Cauchy-Born rule for singlelayer crystalline sheets, and the calculation involves solving the geodesics equation for the deformed surface. In [50] Yang and E developed a simplified approach that approximated the curvature of the tape surface by higher order derivatives of displacements when the deformation is not large and then adopted a generalized Cauchy-Born rule, which takes into account the displacement due to the curvature. In these models, hyperelastic strain energy is used as the continuum model of the system. The above models are strongly nonlinear. Therefore, the numerical solution is done in iteration steps. At each step, the numerical evaluation requires the inner displacement relaxation for the representative volume, and the membrane and bending contributions are tightly coupled. Furthermore, the above methods typically require a crystalline order or periodic setting, which is rarely present in soft matter.

3. Atomistic and continuum modeling of atomistic tape. We first present the setup of our test problem, which has been studied by MD in [12]. Then we introduce the atomistic model and continuum model for $\beta$-tapes.

We mainly focus on the twisting and bending properties of the supramolecular tapes. For the sake of simplicity, we use the united atom model from [12]. The model is tailored to capture essential features of the system, neglecting those details of the molecular structure of amino acids that are unimportant for our purpose.

Consider the following molecular system: $M=60$ peptides (see Figure 2 for an illustration of a single peptide) are placed into a planar, parallel arrangement which forms a flat tape. A single amino acid is represented by three beads (two for the backbone and one for the side chain). The bead denoted as $\mathrm{X}$ stands for the moiety $\mathrm{C}_{\alpha} H-\mathrm{C}^{\prime} \mathrm{O}$, and $\mathrm{Y}$ represents the amine group $(\mathrm{NH})$ on the backbone. The tape has two different sides: one "covered" by $S_{1}$ side chains and another "covered" with $S_{2}$ side chains. The molecular systems are studied by varying both the chiral strength (CS) of the peptide backbone and the solvent affinity asymmetry (SAA) between the Lennard-Jones interaction for the pairs $S_{2}-S_{2}$ and $S_{1}-S_{1}$; the parameters CS and SAA will be explained in more detail later. These peptides can form an atomistic tape via self-assembly $[13,14]$. We can index the beads in this tape by a set of positive integers $\mathcal{I} \subset \mathbb{N}$; the position of the $i$ th bead is $\mathbf{x}_{i} \in \mathbb{R}^{3}$.

3.1. Atomistic modeling of the atomistic tape. The atomistic potential energy of a self-assembling biopolymer has two types of terms: bonded energy terms and nonbonded energy terms. The bonded energy is due to changes in geometry of a single peptide, whereas the nonbonded energy models interaction between peptides in the assembly. We use the simplified atomic potential adopted in [12]. This model is well tested and now actively used for self-assembled aggregation of peptides [13, 14].

$$
E_{a}=\sum_{\text {bonds }} U_{\text {bond }}+\sum_{\text {angles }} U_{\text {angle }}+\sum_{\text {dihedrals }} U_{\text {tors }}+\sum_{i} \sum_{j \neq i} U_{L J}\left(r_{i j}\right)
$$




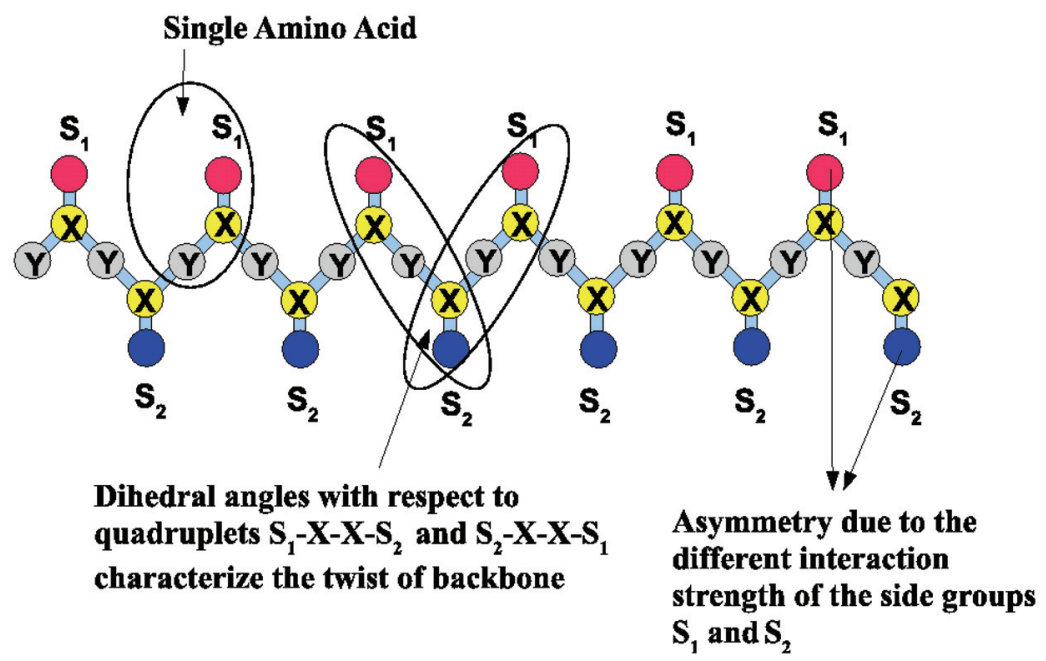

FIG. 2. Geometry of the model peptide.

The numerical values of the energy parameters used in our model, such as those chirality-related parameters, correspond to the ones typically used in atomistic force fields. Their values were chosen to reproduce the experimental range of the macroscopic deformation [12].

The bonded energy consists of the following terms:

1. Bond length potential,

$$
U_{\text {bond }}=\frac{K_{b}}{2}\left(r-r_{e q}\right)^{2},
$$

where $K_{b}=200.0 \mathrm{kcal} / \mathrm{mol} \cdot \AA^{-2}$ and $r_{e q}=2.0 \AA$; the length of each bond is thus restrained toward the equilibrium value via the above harmonic potential.

2. Bond angle potential,

$$
U_{\text {angle }}=\frac{K_{\theta}}{2}\left(\theta-\theta_{e q}\right)^{2},
$$

where $K_{\theta}=40.0 \mathrm{kcal} / \mathrm{mol}$ and $\theta_{e q}$ can take two different values: $\theta_{e q}=120^{\circ}$ for angles centered at $\mathrm{X}$ beads, and $\theta_{e q}=180^{\circ}$ for angles centered at $\mathrm{Y}$ beads. Bond angles defined via triplets $X-Y-X, Y-X-Y$, and $Y-X-S_{1}\left(S_{2}\right)$ are controlled by the above harmonic potential.

3. Dihedral angle potential,

$$
U_{t o r s}=D_{i j k l} \cos \left(3 \alpha-\delta_{i j k l}\right)-G_{i j k l} \cos \left(\alpha-\delta_{i j k l}\right),
$$

where two angles sharing a common bond form a dihedral; for every such set of four atoms, this potential is used to exclude overlap of the first atom and the last atom. In this model, $i j k l$ can be $Y X Y X$ or $X Y X Y$. $D_{i j k l}=G_{i j k l}=$ $1.5 \mathrm{kcal} / \mathrm{mol} ; \delta i j k l=\pi$.

In this paper, we model the intermolecular hydrogen bonding network typical of $\beta$-tape structures by the Lennard-Jones potential as in [12] as follows:

$$
U_{L J}(r)=4 \varepsilon\left[\left(\frac{\sigma}{r}\right)^{12}-\left(\frac{\sigma}{r}\right)^{6}\right]
$$


where $r$ is the distance between two interacting beads and $\varepsilon$ is the interaction strength.

The interaction exists between both backbone beads and side chain beads; see Figure 2. For the interaction involving only the backbone beads $\mathrm{X}$ and $\mathrm{Y}$, we have $\varepsilon=5.0 \mathrm{kcal} / \mathrm{mol}, \sigma=2.0 \AA$ for XX and YY pairs, and $\sigma=3.0 \AA$ for XY pairs. The nonbonded interactions between side chain beads are essential for the self-assembly formation. We define $\varepsilon_{1}$ as the interaction strength for pair $S_{1}-S_{1}$ and $\varepsilon_{2}$ for pair

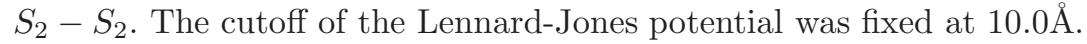

We used the same interaction strength parameter throughout the tape. In practice, the constant $\varepsilon$ in the Lennard-Jones potential can have strong variations even for neighboring atoms.

Once the atomistic potential is given, one can compute the equilibrium configuration of the tape by minimizing the atomistic energy (1). We denote the resulting equilibrium position of bead $i$ as $\mathbf{x}_{i}^{0}$.

The tape will deform from the equilibrium configuration if we impose boundary conditions or apply external loads to it. The deformed configuration of the tape can be computed by minimizing the atomistic energy (1) subject to boundary conditions and external loads. The displacement for the $i$ th bead is denoted by $\mathbf{u}_{i} \in \mathbb{R}^{3}$.

The bending of the $\beta$-tape can be attributed to the following two physical origins at the molecular level:

1. The twist of the backbone: a single peptide (a single strand) in a $\beta$-tape possesses a right-handed twist along the backbone direction, which can be measured by the dihedral angle defined by the quadruplets $S_{2}-X-X-S_{1}$ or $S_{1}-X-X-S_{2}$ within the single strand; see Figure 2. A single peptide monomer with such a right-handed twist assembles with its neighbors at a finite angle. This angle transfers the chirality from the single strands (molecules) to the level of the mesoscopic assembly, e.g., a tape. In the simulation, we take three different values for the dihedral angle parameter: $\delta_{i j k l}=165^{\circ}, 160^{\circ}$, and $155^{\circ}$, which correspond to systems with "chiral strength": $C S=1,2$, and 3 , respectively.

2. The asymmetry of the strength of nonbonded interactions of the $S_{1}$ side and $S_{2}$ side of the tape: we model this assymetry by assigning different values of the parameter $\varepsilon$ for Lennard-Jones interactions on the $S_{1}$ side and $S_{2}$ side of the tape. We vary the parameter $\varepsilon_{2}$ and keep the parameter fixed at $\varepsilon_{1}=1.0 \mathrm{kcal} / \mathrm{mol}$. $\varepsilon_{2}$ can take values from 1.0, 2.0, 3.0, $4.0,5.0,6.0,7.0,8.0$, and $9.0(\mathrm{kcal} / \mathrm{mol})$, which is indexed by the numbers $1,2,3,4,5,6,7,8$, and 9 . We denote this asymmetry parameter by $\mathrm{SAA}=\left(\varepsilon_{2}-\varepsilon_{1}\right) / \varepsilon_{1}$. SAA will be used as an index in the figures in section 5 .

Generally speaking, the backbone twist of an oligopeptide monomer mainly depends on the primary sequence of the oligopeptide. The solvent affinity depends on the chemical nature of the oligopeptide side chains as well as the solvent composition ( $\mathrm{pH}$, ionic strength, etc.). In general, for a given oligopeptide in situ, it is much easier to vary the solvent affinity rather than the backbone twist because one can easily change the strength of peptide-solvent interactions by, for example, changing the $\mathrm{pH}$ value of the solution [3]. Therefore, by switching the $\mathrm{pH}$ value, one can control not only the assembly and disassembly process of the peptides but also the shape of the tapes.

3.2. Continuum modeling of thin elastic structures. Although a most general treatment using hyperelastic constitution law requires the nonlinear coupling of 
bending and membrane energy, the decomposition of elastic energy into membrane and bending (flexural) energy works well in many cases. For example, in [48] a geometric argument was developed to show that the stored energy of a continuous inextensible plate has the following form:

$$
\int_{\Omega} c_{H} H^{2}+c_{K} K d A
$$

where $c_{H}$ and $c_{K}$ are material coefficients, $H$ is the mean curvature, and $K$ is the Gaussian curvature. In our case, we adopt the Saint Venant-Kirchhoff model analogous to (6) for the elastic tape to achieve a trade-off between accuracy and efficiency [7] of the numerical model.

Under the assumption of inextensibility (see also discussions in section 2.2), the elastic energy is purely bending, and the bending energy can be written in a compact form by using the shape operator [53] described as follows. Let $\Omega$ be a smooth surface which can be seen as the middle surface of a shell or a surface of a membrane of zero thickness, where $\mathbf{n}$ and $\mathbf{T}$ are unit normal and unit tangent to surface $\Omega$, respectively. The shape operator $S$ of $\mathbf{n}$ acting on $\mathbf{T}$ can be defined as $S_{\mathbf{n}}(\mathbf{T}):=-\partial_{\mathbf{T}} \mathbf{n}$ (for simplicity, denoted below by $S$ ). Suppose that $Q=\nabla u$ is the gradient of surface deformation. The difference between $Q^{T} S Q$ and $S$ is of higher order and can be neglected for small strains. For a Kirchhoff-Love inextensible shell of thickness $2 h$, the Koiter bending energy has the following form [53]:

$$
\begin{aligned}
U & =\frac{D}{2} \int_{\Omega}\left[\nu\left(\operatorname{tr}\left(Q^{T} S Q-S_{0}\right)\right)^{2}+(1-\nu) \operatorname{tr}\left(\left(Q^{T} S Q-S_{0}\right)^{2}\right)\right] d \sigma \\
& \simeq \frac{D}{2} \int_{\Omega}\left[\nu(\operatorname{tr}(\Delta S))^{2}+(1-\nu) \operatorname{tr}\left((\Delta S)^{2}\right)\right] d \sigma \\
& =D \int_{\Omega}\left[(1+\nu)\left(H-H_{0}\right)^{2}+(1-\nu)\left((\Delta A)^{2}+4 A A_{0} \sin ^{2} \theta\right)\right] d \sigma
\end{aligned}
$$

where $S_{0}$ is the shape operator for the undeformed surface, $\Delta S:=S-S_{0}, A=$ $\sqrt{H^{2}-G}, H$ is the mean curvature, $G$ is the Gaussian curvature, and $H_{0}$ is the mean curvature for the undeformed surface, also called spontaneous curvature [49]. $\theta$ is the angle of principal axes between deformed and undeformed surfaces. Note that $H$ is the trace and $G$ is the determinant of the shape operator $S$. The bending constant $D$ is given by $D=2 h^{3} E /\left[3\left(1-\nu^{2}\right)\right]$, where $E$ is Young's modulus, and $\nu$ is Poisson's ratio. In fact, for our supramolecular tape, it is not immediately clear how to define $h[36]$ because we have different types of atoms in our monomers. In the work of [53], the following formula for a single layer atomic tape was introduced:

$$
\left.E_{1}=\int_{\Omega}\left[\alpha_{1}\left(H-H_{0}\right)^{2}+\beta_{1}\left((\Delta A)^{2}+4 A A_{0} \sin ^{2} \theta\right)\right]+\gamma_{1}\right] d \sigma
$$

with effective parameters $\alpha_{1}, \beta_{1}$, and $\gamma_{1}$ to be determined from the atomistic model.

An alternative of the above form (8) is the so-called Helfrich energy functional [31]:

$$
E_{2}=\int_{\Omega} \frac{1}{2}\left[\alpha_{2}\left(H-H_{0}\right)^{2}+\beta_{2} K+\gamma_{2}\right] d \sigma .
$$

It can be seen as an approximate shell model and is frequently used in computer graphics and membrane physics, although it is usually used to model fluid membrane under this context [31]; that is, $\theta=0$ in formula (8).

In the biological literature $[4,12]$, the following one-dimensional model for anisotropic elastic rod is used as a coarse-grained model. It is assumed that the tape 
has fixed width and length; then the energy is given by

$$
U=\int_{0}^{L}\left[\alpha_{3}\left(k-k_{0}\right)^{2}+\beta_{3}\left(\eta-\eta_{0}\right)^{2}+\gamma_{3}\right] d s
$$

with $k$ and $\eta$ the curvature and the torsion of the centerline of the tape, respectively, whereas $k_{0}$ and $\eta_{0}$ are the curvature and the torsion for their equilibrium counterparts. The value of curvature $k$ and torsion $\eta$ can be calculated numerically by the method in [35].

The assumption of model (10) is that the conformation of the tape is only determined by its centerline and a direction vector orthogonal to the centerline. The deformation energy functional of a membrane can be decomposed into twisting, bending, and splay modes. We assume the length and the width of our tape are constant, the splay deformation is negligible, and that one needs to consider only the twisting and the bending energy. In this paper, we will test all three models and show that although the one-dimensional model is simple to use, it has limited accuracy. Note that the one-dimensional model cannot deal with the situation when the peptide consists of different amino acids.

Remark 3.1. In a recent work [36], the authors derived the bending constant for a graphene monolayer from an atomistic model where only bonded interactions are present. If the equilibrium configuration is flat, an analytical formula of the bending modulus can be derived from the knowledge of the atomistic potentials. Any atomic model with only the bonded length potential (nearest neighbor interactions) would lead to a zero bending modulus of the monolayer, which is nonphysical.

3.3. Discretization for the continuum energy of the atomistic tape. Both the bending energy $E_{1}$ derived from shell theory (8) and the Helfrich bending energy $E_{2}(9)$ involve the computation of curvature-based quantities: mean curvature, Gaussian curvature, principal directions, and principal curvatures.

The atomistic tape can be viewed as a surface $S$. See Figure 2; the collection of all beads $Y$ form a set of points that is interpolated by a smooth surface $S$. We next approximate the surface $S$ by a piecewise flat surface $S_{h}$ composed of triangles of size $h \gg \delta ; \delta$ is the atomistic spacing. We call the triangulation $\mathcal{T}$. The vertices of these triangles are also beads. See Figure 3 for an illustration.

We index the $Y$ beads of the tape surface $S$ by $Y_{i, j}$, where $i$ denotes its position in the oligopeptide sequence and $j$ is the monomer number of the oligopeptide in the tape. For the tape described at the beginning of section $3, i \in I=\{1, \ldots, 12\}$, $j \in J=\{1, \ldots, 60\}$. Similarly, we index the vertices $P$ of the piecewise flat surface as $P_{i c, j c}$. We denote $I c$ as the set of indices $i c$, and $J c$ is the set of indices $j c$. The triangle $P_{i c, j c+1} P_{i c, j c} P_{i c+1, j c}$ is a typical triangle of $\mathcal{T}$.

An atomistic displacement field $u^{A}$ introduced in section 3.1 can be projected from the atomistic scale $\delta$ to the coarse displacement field $u^{C}$ on the piecewise flat triangulated surface $S_{h}$. For simplicity, we use piecewise linear interpolation as follows. At the vertices $P$ of the triangulation, we have

$$
u^{C}(P)=u^{A}(P),
$$

and at the interior of each triangle, the displacement $u^{C}$ is the linear interpolation of the displacements of the three vertices of that triangle.

To calculate the curvature for this piecewise flat triangulated surface, we can use polynomial reconstruction and analytical evaluation. However, this often leads 


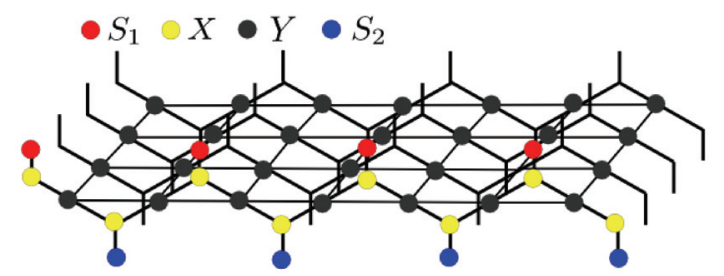

The tape surface formed by peptides in Figure 2

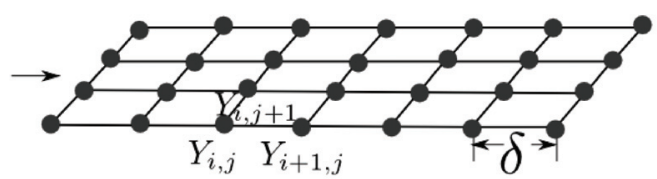

Surface $S$ determined by beads $Y$

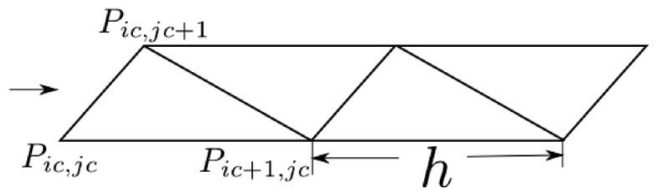

Piecewise flat triangulated surface $S_{h}$

FIG. 3. From atomistic tape to piecewise flat triangulated surface. To improve visibility, all-beads representation is only shown for the peptide in the front of the top figure. For other peptides, we only show $Y$ beads.

to spurious oscillation (artifacts of numerics) and is computationally expensive [38]. Alternatively, we can use discrete differential geometry techniques to calculate discrete curvatures $[38,47]$. These techniques are more robust and more efficient. In this way, we can go directly from atomistic representation to a discretized problem, and we do not need to use continuum description as an intermediate step. For completeness, we will introduce the technique to compute discrete curvature in the following section.

3.3.1. Discrete curvatures of triangulated surface. For the definition of discrete curvature, we will follow [38, 47, 53]. For a triangulated surface, the discrete curvatures - mean curvature, Gaussian curvature, principal curvatures - as well as principal directions are defined on the vertices of the triangulation. 1-ring neighbors of a vertex are all the vertices which are connected to the vertex by one edge. The discrete curvatures depend only on the positions of the vertex itself and its 1-ring neighbors. We assume that all the triangles are nonobtuse; for general situations, please refer to [38].

Let $\Omega$ be a surface in $\mathbb{R}^{3}$; suppose that $H$ is the mean curvature of $\Omega$, and $G$ is the Gaussian curvature of $\Omega . \kappa_{1}$ and $\kappa_{2}$ are two principal curvatures with their associated principal directions $e_{1}$ and $e_{2}$, respectively. We have $H=\frac{\kappa_{1}+\kappa_{2}}{2}$ and $G=\kappa_{1} \kappa_{2}$. It is often more convenient to work with the mean curvature vector defined by $\mathbf{L}(P):=$ $2 H(P) \mathbf{n}(P)$ at a point $P$. See Figure 4(a) for an illustration. $\mathbf{L}$ is also known as the Laplace-Beltrami operator for the surface $\Omega$.

It is not straightforward to define quantities like tangent plane or normal vector for a vertex on a triangulated surface as we can do for a smooth surface. Alternatively, one can define geometric quantities of the triangulated surface at a vertex as spatial averages around this vertex. For example, the unit normal vector at vertex $P$ can be defined as the normalized spatial average of normal vectors $\frac{1}{A(P)} \int_{A(P)} \mathbf{n} d \sigma$, and the 


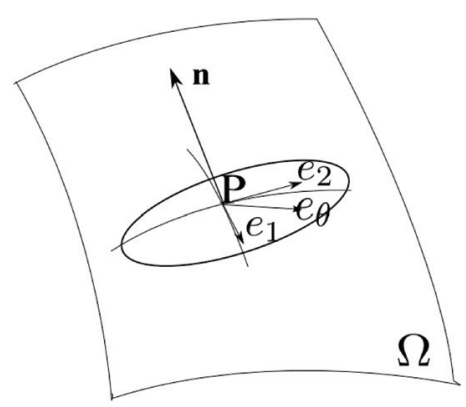

(a) Principal directions $e_{1}$ and $e_{2}$ associated with two principal curvatures $\kappa_{1}$ and $\kappa_{2}$

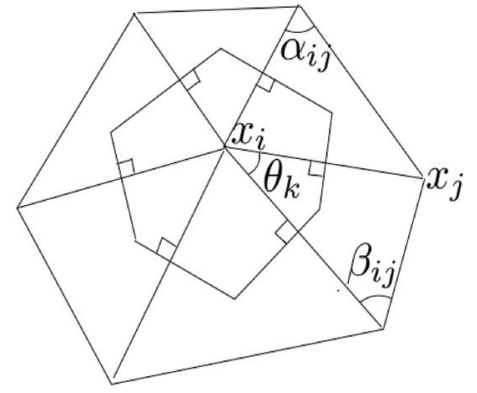

(b) Voronoi cell around a vertex which can be used to define discrete curvature

FIG. 4. Definition of discrete curvature.

mean curvature vector $\mathbf{L}$ at a vertex $P$ can be defined as

$$
\mathbf{L}(P)=\frac{1}{A(P)} \int_{A(P)} \mathbf{L}(x) d \sigma,
$$

where $A(P)$ is a properly chosen area associated with $P$. For example, if all the triangles are nonobtuse, $A(P)$ can be chosen as the Voronoi cell around $P$ (Figure $4(\mathrm{~b}))$.

Analogous to its smooth counterpart, the discrete mean curvature vector $\mathbf{L}$ defined at vertex $P=\mathbf{x}_{i}$ is the first variation of the area $A(P)$, which can be expressed as

$$
\begin{aligned}
\mathbf{L}(P) & =2 H(P) \mathbf{n}(P)=-\nabla_{P} A(P) \\
& =\frac{1}{2 A(P)} \sum_{j \in N_{1}(i)}\left(\cot \alpha_{i j}+\cot \beta_{i j}\right)\left(\mathbf{x}_{i}-\mathbf{x}_{j}\right),
\end{aligned}
$$

where $\alpha_{i j}$ and $\beta_{i j}$ are the two angles opposite to the edge $\left(\mathbf{x}_{i}, \mathbf{x}_{j}\right)$ and $N_{1}(i)$ is the set of 1-ring neighbors of the vertex $P=\mathbf{x}_{i}$ as show in Figure 4(b).

Gaussian curvature at the vertex $P$ can be calculated by the so-called angle deficit formula which keeps the Gauss-Bonnet theorem true, namely,

$$
K(p) A(P)=\int_{A(p)} K d \sigma=2 \pi-\sum_{i} \theta_{i} .
$$

Here the angles $\theta_{i}$ are the angles at $P$ of the triangles meeting there.

Once the mean curvature and Gaussian curvatures are defined, principal curvatures can be easily computed by

$$
\kappa_{1,2}=H \pm \sqrt{H^{2}-G} .
$$

To find the principal directions, first we use the osculating circle approximation [21] to define the normal curvature in the direction $\mathbf{x}_{i} \mathbf{x}_{j}$ for each 1-ring neighbor $\mathbf{x}_{j}$. The osculating circle passes through $\mathbf{x}_{i}, \mathbf{x}_{j}$, with $\mathbf{n}$ as its normal direction at $\mathbf{x}_{i}$, and the normal curvature $\kappa_{i, j}$ is the inverse of the osculating circle radius as follows:

$$
\kappa_{i, j}=2 \frac{\left(\mathbf{x}_{i}-\mathbf{x}_{j}\right) \cdot \mathbf{n}}{\left\|\mathbf{x}_{i}-\mathbf{x}_{j}\right\|^{2}} .
$$


Let us introduce the curvature tensor $C$ as

$$
C=\left(\begin{array}{ll}
a & b \\
b & c
\end{array}\right) .
$$

Let $d_{i, j}$ be the unit vector projection of $\mathbf{x}_{i} \mathbf{x}_{j}$ on the tangent plane; we have the following quadratic relation:

$$
d_{i, j}^{T} C d_{i, j}=\kappa_{i, j}
$$

In general (18) is an overdetermined relation because $C$ has only three unknowns, but since we have six triangles meeting at $P$ for a regular triangulation, $C$ can be obtained through a least square fitting, and principal directions $e_{1}$ and $e_{2}$ are eigenvectors of $C$.

3.4. Inextensible constraint and boundary conditions. Representing the surface by a piecewise flat triangulation $\mathcal{T}$, we can express the bending energy by either (8) or (9) through a summation over vertices,

$$
E_{B}=\sum_{P} f\left(H(P), G(P), e_{1}(P), e_{2}(P)\right) A(P)
$$

where $f$ is an energy form in terms of the curvature-related quantities $H(P), G(P)$, principal direction $e_{1}(P)$, and $e_{2}(P)$. In actual simulations, we will choose $f$ to be one of the functionals (8) or (9).

We need to find the minimizer of the above discretized energy up to appropriate boundary conditions and inextensibility condition.

The inextensibility condition is enforced as a penalty formulation. We write the total energy as a sum of membrane and bending energy [30],

$$
E_{\text {total }}=E_{M}+k_{B} E_{B}
$$

where $k_{B}$ is the bending or flexural stiffness, and $E_{M}$ is the penalized membrane energy defined as follows. We decompose $E_{M}=k_{L} E_{L}+K_{A} E_{A}$, where the shearing energy $E_{L}$ measures local change in length as follows:

$$
E_{L}=\sum_{e}\left(1-\|e\| /\left\|e_{0}\right\|\right)^{2}\left\|e_{0}\right\|
$$

where $e$ is the edge of the deformed triangle, $e_{0}$ is the edge of the undeformed triangle, and $\|e\|$ and $\left\|e_{0}\right\|$ stand for lengths of the edges. The summation is taken over all edges in the piecewise flat surface $\mathcal{T}$.

The stretching energy $E_{A}$ measures local change in area as follows:

$$
E_{A}=\sum_{A}\left(1-\|A\| /\left\|A_{0}\right\|\right)^{2}\left\|A_{0}\right\|
$$

where $\mathrm{A}$ is the deformed triangle, $A_{0}$ is the undeformed triangle, and $\|A\|$ and $\left\|A_{0}\right\|$ stand for areas of a triangle. The summation is taken over all triangles in the piecewise flat surface $\mathcal{T}$.

The inextensibility is achieved by choosing sufficiently large stiffness constants $k_{L}$ and $k_{A}$. Since we cannot model the inextensibility exactly, in practice we choose an increasing sequence of stiffness constants $k_{L_{n}}$ and $k_{A_{n}}$ to achieve convergence of energy [49]. 
Remark 3.2. In general, the penalty on the membrane deformation introduces complicated numerical coupling between the bending and membrane energies. There is no analytical result that guarantees the convergence to a minimizer. We will show that for our numerical experiments, the membrane energy is indeed small compared to the bending energy, and therefore, the minimization of the penalized energy converges; see also Remark 5.4.

Boundary conditions on $\partial \Omega$ are enforced as constraints over positions of boundary vertices. The Dirichlet boundary condition is straightforward. For a natural boundary condition, we calculate the force numerically as the gradient of the energy and enforce the zero force condition as a nonlinear constraint.

4. Atomistic-to-continuum (AtC) modeling by the energy matching method. We will develop a global energy matching method for the self-assembly system. The main issues of these systems are the following: First, they are noncrystalline, and therefore, the lattice is not periodic; second, they are generally highly heterogeneous on both micro- and macroscales with nonseparable scales. Third, the energy is nonlinear.

In principle, the AtC transition could be done by taking the homogenization limit in the atomistic model, as lattice spacing tends to 0 (for a review of rigorous results on AtC limit, see, e.g., $[17,18,20,24]$ ). However, this problem is presently intractable because of the complex form of the atomistic energy (1); in particular, the constant $\varepsilon$ in the Lennard-Jones potential can have strong variations even for neighboring atoms. To the best of our knowledge, we are not aware of existing AtC methods which can accurately handle all these problems. In this paper, we deal with this difficulty by introducing an energy-based method. The main idea is to postulate the macroscopic continuum energy and match it with the atomistic energy. More discussions on comparing our method with the existing ones are presented in the next subsection.

Our goal is to obtain effective elastic properties for the hierarchical supramolecular structures (see Figure 1). In this paper we restrict ourselves to numerical evaluation of effective constants for the self-assembled $\beta$-tapes described in section 2.1.

We postulate a specific macroscopic (continuum) form of the energy and determine material constants in this energy by enforcing the match between that continuum energy (20) (with coefficients defined for $E_{1}$ in (8), $E_{2}$ in (9), and $E_{3}$ in (10)) and the discrete energy $E_{a}(1)$. Theoretically, it should be done for all possible displacement fields. However, in practice, the key is to choose the minimal amount of special representative fields as follows:

$$
E_{a}=E_{\text {total }} \sim k_{B} E_{B}
$$

Equation (23) has unknowns $k_{B}$ and coefficients in the energy forms (8), (9) or (10). ${ }^{1}$ The unknowns are found by substituting discrete displacement fields that minimize (1) under appropriate boundary conditions into (23).

Let $u$ be the displacement field for the discrete energy (1). We find the minimizers of (1) by imposing Dirichlet boundary conditions $u=f(x)$ on the boundary of the tape and varying $f(x)$. For fixed $f(x)$, we choose a minimizing sequence and use the standard stopping criteria numerically. For example, in the Helfrich energy (9), we have three different unknown effective material constants. Therefore, we choose three different functions $f(x)$ that correspond to three different twists. For each value of $f$, we find the minimizer numerically by the BFGS quasi-Newton method [40].

\footnotetext{
${ }^{1} k_{B}$ can be absorbed into coefficients of the specific energy form.
} 
In general the minimizer is not unique, and in actual numerical procedures, instead of finding a global minimizer, we numerically find approximate physically meaningful local minimizers.

Remark 4.1. In the equality (23), due to the inextensibility condition (see section $3.4), E_{\text {total }}$ can be approximately represented as $k_{B} E_{B}$ up to an additive constant which is set to 0 in (23) for simplicity. In practice, this additive constant is also an unknown parameter for the continuum model.

Remark 4.2. If the medium is heterogeneous (noncrystalline), then the projection of the continuum model on the coarse piecewise flat surface (with $N$ triangles) is characterized by $N \times m$ unknown parameters. However, $m$ precomputed (global atomistic) solutions are still sufficient to determine those $N \times m$ unknown parameters by enforcing the energy matching principle in each triangle.

Remark 4.3. The concept of precomputed solutions can be seen as an analogue of the periodic cell problems $[15,33]$ for the homogenization of partial differential equations. The advantage of solving precomputed solutions numerically rather than solving directly the full original problem comes when, e.g., we need to solve for many different loads or boundary conditions. For more detailed discussions regarding precomputed solutions under the context of the numerical homogenization for partial differential equations, see [34].

Our method can be summarized in the following steps:

1. A continuum energy with $m$ unknown parameters is postulated in one of the forms: $m=3$ for (8), (9), and (10).

2. We construct a piecewise flat surface $S_{h}$ from coarse triangles; the size of triangles $h$ is much larger than the atomistic scale $\delta$ (refer to Figure 3).

3. A discrete representation (19) of the continuum energy is obtained on the surface $S_{h}$.

4. $m$ representative and independent solutions $u^{A, i}, i=1, \ldots, m$ are computed by minimizing the atomistic model (1) with appropriate boundary conditions.

5. From discrete solution $u^{A, i}$, we construct a piecewise linear function $u^{C, i}$ as described in section $3.3 ; u^{C, i}$ is viewed as an approximate continuum minimizer on the piecewise flat surface.

6. Obtain $m$ equations by matching the atomistic energy of each solution $u^{A, i}$ with the continuum energy of corresponding function $u^{C, i}$ defined on the piecewise flat surface $S_{h}$ :

$$
E_{a}\left(u^{A, i}\right)=E_{\text {total }}\left(u^{C, i}\right)
$$

7. Equation (24) determines $m$ unknown material constants in the energy (8), (9), or (10).

The rationale behind the method in the context of AtC upscaling lies in a modification of the Cauchy-Born rule. For crystalline materials, the Cauchy-Born rule $[19,28,25,22]$ states that at fine scales, displacements are essentially linear. The Cauchy-Born rule no longer holds for noncrystalline materials; however, our method is based on the rule (conjecture) that at fine scales, displacement solution set can be approximated by linear space of low dimension. In the following, we will call this rule the rule of low-dimensional representation. Namely, at fine scales, all displacements can be approximated (in energy norm) by the linear span of a few precomputed displacements. In our case, the $m$-dimensional subspace is the span of $m$ solutions $u^{A, i}$ by $m$ different boundary conditions.

How to choose the $m$ precomputed solutions? According to the rule of lowdimensional representation for heterogeneous materials, any set of $m$ precomputed 
solutions should be sufficient to approximate the solution space of the atomistic model provided that those solutions are linearly independent on each triangle of the coarse mesh. Two different sets of $m$ precomputed solutions will lead to two distinct continuous model parameters; however, according to the new rule, the error between those parameters will be proportional to the size of the coarse mesh on which the continuous model is projected. This being said, a given set of $m$ precomputed solutions may lead to an ill conditioned system of equations for the continuous model's unknown parameters (and hence to a large error constant associated with the size of the coarse mesh). If the resulting system is ill posed, we may need additional solutions and solve the system in a mean squared sense. Ideally, one would like to choose the minimum number of solutions leading to a well conditioned system. Although there is no general recipe on how to choose the $m$ precomputed solutions in order to obtain well conditioned systems, one can use simple physical considerations as a guide. For instance, for the model considered in this paper, twisting and bending are the most important physically independent modes of deformation. Therefore, we can choose the boundary condition by twisting or bending the equilibrium configuration. For scalar elliptic equations a typical choice of precomputed solutions consists of harmonic coordinates $[41,16]$. For elasticity equations, one can use harmonic displacements with linear Neumann or Dirichlet boundary conditions [34, 16].

4.1. Novelty and advantage of the method. The global energy matching method works for general atomistic configurations, not necessarily periodic or homogeneous. Most biological assemblies are of noncrystalline nature. However, most previous AtC methods were constrained by crystalline order or periodic structure $[27,7]$ and, therefore, cannot be directly applied to the "soft matter" system. On the contrary, the energy matching method does not require crystalline order and works for general microstructures such as nonstructured/amorphous systems.

The global energy matching method is based on a completely different reasoning from previous AtC methods. The underlying mathematical rationale for the energy matching method is the existence of a low-dimensional approximating functional subspace to the atomistic system, which can be viewed as a particular case of the thin subspace idea introduced in [16], while local energy-based methods, such as the Cauchy-Born rule, are often based on an asymptotic analysis.

First results on energy matching methods can be traced back to the works $[8,9,46]$. In these works, the local energy matching principle, also called the recovery method, was introduced for solving large systems of lattice equations. The local (restricted to subdomains of mesoscopic size) energy matching principle is used as a preconditioner for fine scale equations rather than for homogenization as in the present paper. The main novelty here is that our method is global and it is better suited for inhomogeneous media such as supramolecular aggregates considered in this paper. In parallel with our work from AtC elasticity, similar energy matching techniques have been applied to the coarse graining of the linear elasticity equation [34]. ${ }^{2}$ In this paper, we develop an approach which allows us to compute an effective modulus and approximate displacements from nonlinear atomistic models to a continuum nonlinear elasticity model (geometric nonlinearity due to the curvature). While other approaches assume a specific form of the energy, our approach allows us to select the

\footnotetext{
${ }^{2}$ Moreover, the method proposed in subsection 1.3 of [41] is equivalent to the energy matching principle applied to the upscaling of scalar elliptic partial differential equations and proven to be accurate with arbitrary rough coefficients.
} 
best form for effective continuum energy from several candidates; e.g., in this paper we can choose from (8)-(10).

5. Numerical results. We perform numerical experiments for the supramolecular tapes. Sixty peptides $(M=60)$ are placed into a planar parallel arrangement which forms a flat tape. We compute the equilibrium configuration first by minimizing the potential energy at temperature $T=0 K$. Once we have the equilibrium configuration, we can calculate geometric quantities such as curvatures and material parameters in (8), (9), and (10) and obtain the continuum model using our energy matching method. The atomistic simulations are done by NAMD [43], the continuum simulations are done in Matlab, and the minimization is done by the BFGS quasi-Newton method [40].

5.1. Structural transition and mean curvature. The parameters SAA and CS, introduced in section 3.1, reflect the physical origins of the deformation of the atomistic tape. In Figure 5, we show the equilibrium configuration of the tape with parameters $\mathrm{SAA}=5, \mathrm{CS}=1$, and the discrete geometrical quantities such as mean

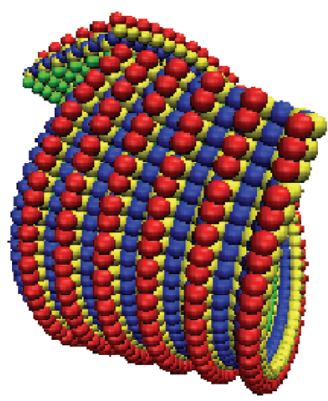

(a) Equilibrium configuration, $\mathrm{SAA}=5, \mathrm{CS}=1$

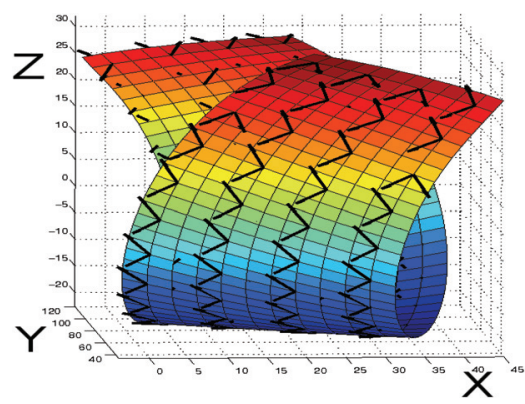

(c) Principal directions $e_{1}$ and $e_{2}$ associated with two principal curvatures $\kappa_{1}$ and $\kappa_{2}$

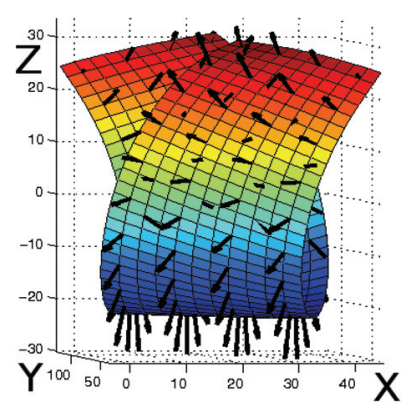

(b) Mean curvature vector

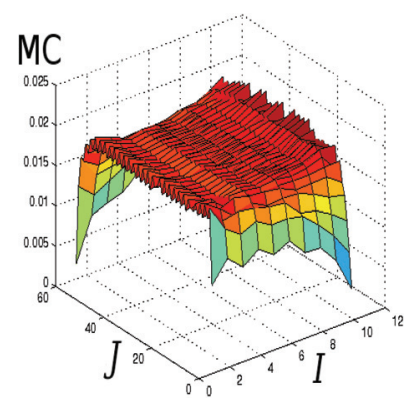

(d) MC: Mean curvature, $I$ : index for peptide sequence, $J$ : index for monomer number, see section 3.3

FIG. 5. Equilibrium configuration and discrete geometric quantities. 
curvature vector, principal directions, and mean curvature. Notice that except at the boundary, the value of mean curvature is almost constant. This is not surprising since the peptide in our problem is homogeneous. The drop of the mean curvature at the boundaries is due to the finite size of the system and should be negligible for systems with large size.

In [12], the authors found that when the asymmetry of the side chains (SAA) or twist of the peptide backbone (CS) increases, the equilibrium configuration of the $\beta$-tape switches from helicoid to cylindrical shape. This can be seen in Figure 6 .

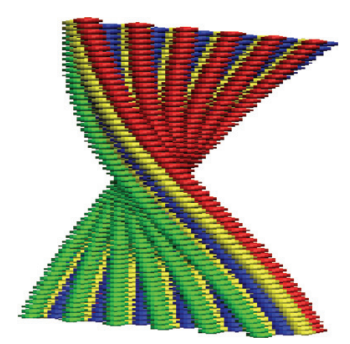

(a) $\mathrm{SAA}=1, \mathrm{CS}=1$

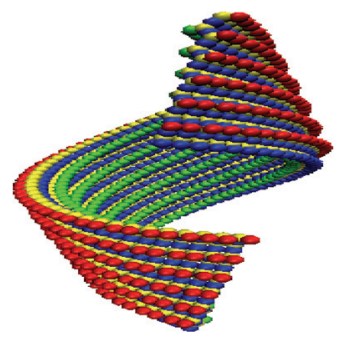

(c) $\mathrm{SAA}=5, \mathrm{CS}=3$

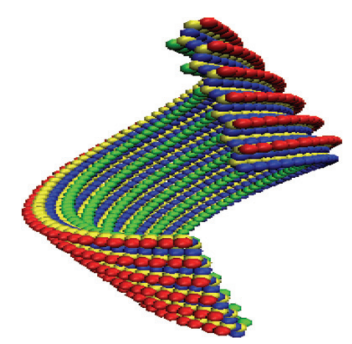

(b) $\mathrm{SAA}=5, \mathrm{CS}=1$

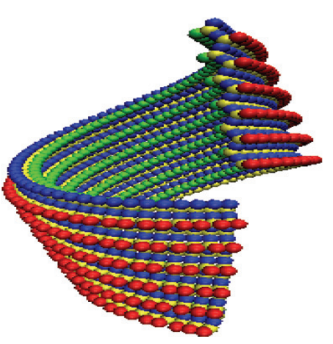

(d) $\mathrm{SAA}=9, \mathrm{CS}=1$

FIG. 6. Equilibrium configuration with different atomistic parameters.

Corresponding to the transition of structures, the mean curvature increases as the asymmetry of the side chains grows and as the peptide backbone becomes more twisted. We compute the mean curvatures for equilibrium configurations as an indicator for bending with respect to parameters SAA, ranging from 0 to 8, and CS, ranging from 1 to 3 (Figure 7).

5.2. Twisted tape: Verification of the global energy matching method. We use this example to test the accuracy of the global energy matching method. Consider a deformed tape with two shorter sides twisted and then clamped at fixed position. The two shorter sides will be twisted by $n^{\circ}$ from the equilibrium configuration, $n=1, \ldots, 10$. By matching the energy, we can obtain the parameters of the elastic model. Here we will test all the models: shell bending energy $E_{1}$ in (8), the Helfrich bending energy $E_{2}$ in (9), and one-dimensional energy $E_{3}$ in (10). We used the atomistic simulation for $n=0,1,2,3$ to generate a training set and obtain the parameter set. 


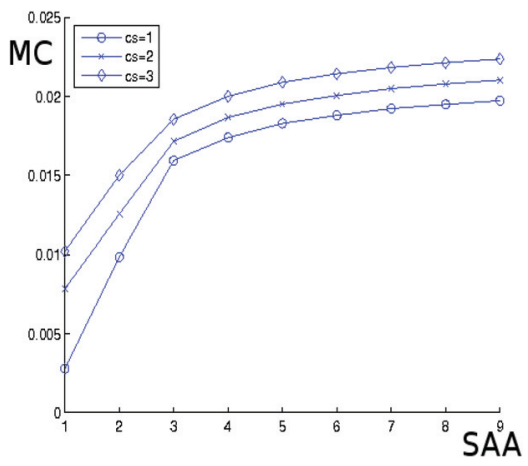

FIG. 7. Mean curvature (denoted by $M C$ in the figure) with respect to side chain asymmetry (SAA) and backbone twist (CS).

Figure 8 shows the configuration from the steered (constrained) MD simulation [43] and continuum simulation using the Helfrich bending energy (9) for $n=10$. Note that the continuum simulation is done on the piecewise flat triangulated surface with typical size $h=2 \delta$, where $\delta$ is the typical distance of neighboring amino acids. The resulting configurations of the twisted tape are visually close to each other.

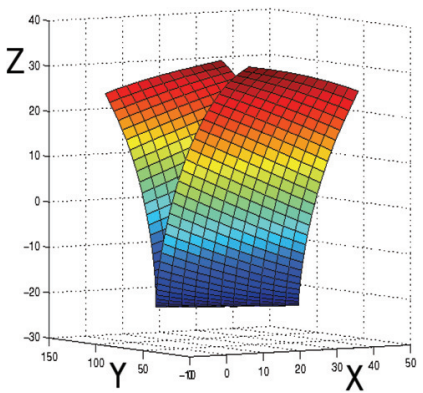

(a) Equilibrium, MD simulation

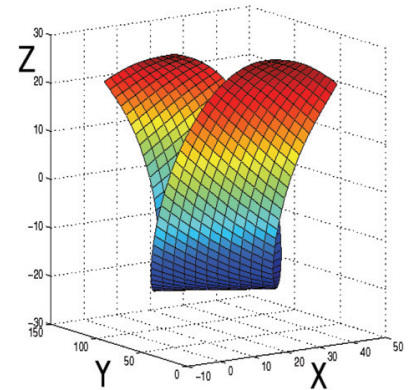

(b) Deformed, MD simulation

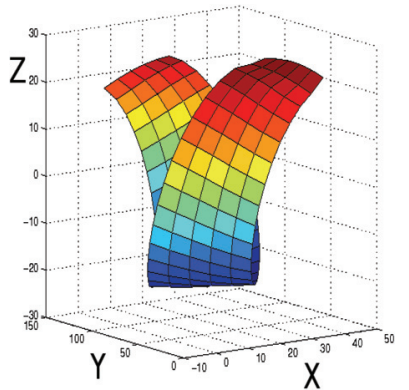

(c) Deformed, elastic model

FIG. 8. Atomistic tape with shorter sides twisted $10^{\circ}$.

Remark 5.1. The numerical computation of the elastic model, in particular, the geometric quantities such as curvatures, requires the triangulation $\mathcal{T}$. But for visualization purposes, we only need to plot the position of vertices, and we do not draw the triangulation in Figure 8 and the following figures.

Remark 5.2. Here we briefly discuss the computational cost of the global energy matching method. At the training stage, we need to compute $m$ displacements by atomistic simulation. Once this is done, we can use the elastic model for further simulations. For our test problem, the degree of freedom for atomistic simulation is $60 \times 12 \times 3 \times 3=6480$, and the degree of freedom for the elastic model in Figure 8(c) is 540. The computational time for the atomistic simulation on a desktop computer with an Intel Core i7-870 2.93GHz processor is about 3 hours, and for the continuum simulation, it is about 20 to 30 minutes. 
Remark 5.3. For the one-dimensional model, we only calculate the energy for the atomistic simulation result. Whereas for the two-dimensional model $E_{1}$ and $E_{2}$, once we have obtained the parameters, we use the energy to compute the respective energy minimizers subject to different boundary conditions.

Remark 5.4. We have checked the membrane energy for the atomistic tape for all twist angles: it is about $1 \%$ of the bending energy. We actually impose this constraint also in the numerical minimization of the penalized energy.

In Figure 9, the deformation energy is plotted against twist angle $n$ for all the three continuum models, with $m=4$. We can see that the two-dimensional shell model is most accurate. Surprisingly, the one-dimensional rod model is better than the Helfrich model. The reason is that our deformation here still retains symmetry; therefore, the one-dimensional model is appropriate. Another reason could be that the Helfrich model does not take into account the deformation of the principal axis.

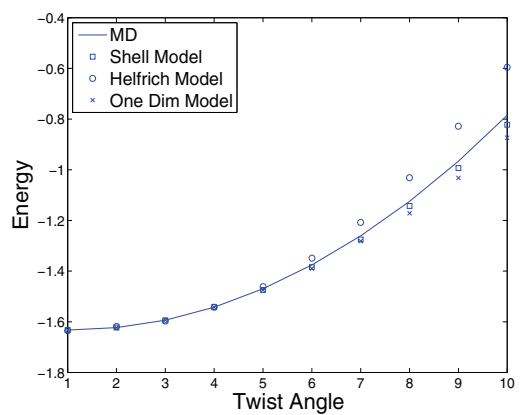

FiG. 9. Energy (E) vs. twist angle.

Now we change the number of displacements in the training set; namely, we use $m=3, \ldots, 8$ displacements to obtain the parameters and use these parameters to generate the elastic energy $E_{1}, E_{2}$, and $E_{3}$. Then we measure the error of elastic model by

$$
e(m)=\max _{\text {twist } n=0, \cdots, 10}\left|E_{a}-E_{i}\right| .
$$

We present Table 1 for $e(m)$.

TABLE 1

Errors of different models in terms of energy.

\begin{tabular}{|c||c|c|c|c|c|c|}
\hline$m$ & 3 & 4 & 5 & 6 & 7 & 8 \\
\hline$E_{1}$ & 1.9411 & 0.1899 & 0.1122 & 0.0513 & 0.0293 & 0.0094 \\
\hline$E_{2}$ & 0.0122 & 0.0375 & 0.0138 & 0.0077 & 0.0041 & 0.0057 \\
\hline$E_{3}$ & 0.0572 & 0.0888 & 0.0066 & 0.0086 & 0.0132 & 0.0032 \\
\hline
\end{tabular}

From Table 1, we see that as more displacements are added to the training set, more accurate results can be obtained. The shell model $E_{2}$ is most accurate; even using the minimum required number of training displacements $m=3$, we get a small error compared with atomistic simulation. As a one-dimensional rod model, $E_{3}$ is also a good option. Although it cannot reproduce the geometry of the two-dimensional tape, it can reproduce the energy with relatively lower computational cost. 
5.3. Using obtained parameters to simulate longer molecular tape. Notice that from Figure 5, the mean curvature of the atomistic tape is almost constant except at the boundary. Since there is no long-range interaction between different parts of the tape, we can use the parameters computed for the tape of $M=60$ peptides to calculate the equilibrium configuration for a self-assembled tape with $M=120$ peptides, which is reminiscent of the so-called cell problem in homogenization. Namely, we can connect two tapes consisting of $M=60$ peptides together. We use the following compatibility condition at the common sides which join two tapes together: the normal vectors computed from each tape coincide at the common sides.

From Figures 10 and 11, we can see that the configurations computed by atomistic simulation and the continuum method are visually similar, and the mean curvature is almost constant in the interior of the tape.

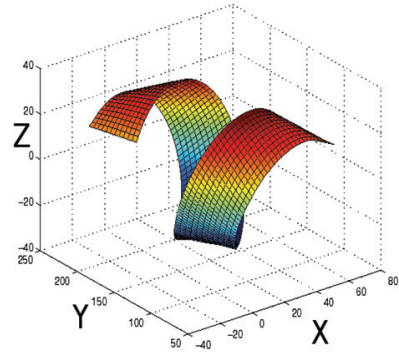

(a) equilibrium configuration, atomistic simulation

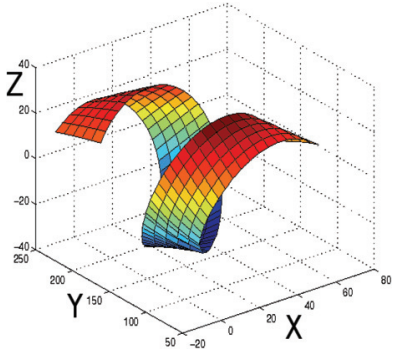

(b) equilibrium configuration, elastic model

FIG. 10. Tape of $M=120$ peptides.

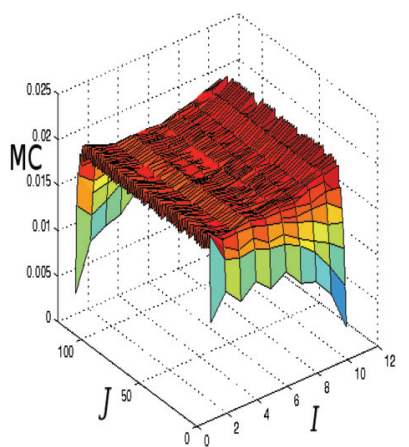

(a) atomistic simulation, $I$ : index for peptide sequence, $J$ : index for monomer number, see section 3.3.

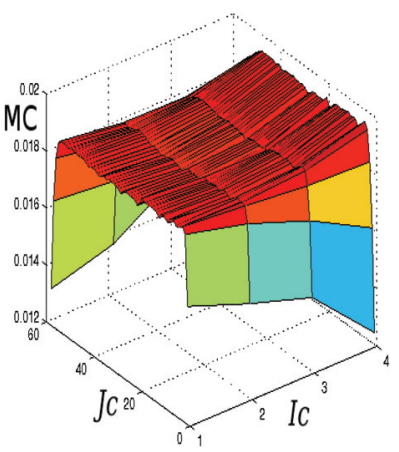

(b) elastic model, Ic and $J c$ : index for the vertices of triangulated surface, corresponding to $I$ and $J$ respectively, see section 3.3 .

FIG. 11. Mean curvature (MC): tape of $M=120$ peptides. 
6. Conclusion. In this work, we propose a way to determine macroscopic characteristics from atomistic parameters for an important class of nanoobjectssupramolecular aggregates. On a set of test systems, peptide self-assembling $\beta$-tapes, we showed how to obtain curvature elasticity models from an atomistic model by the energy matching method. It is important to note that this method can be applied to many kinds of soft matter systems (polymer assemblies, peptide/protein aggregates, amorphous nanocomposites, etc.) because the energy matching method requires neither crystalline order nor periodic setting. We used the discrete geometry tool to represent the curvature-based elastic energy.

The numerical tests show that the method is robust with respect to changes in the definitions of the energy functionals (at least for this class of supramolecular objects): numerical experiments indicate that reasonable results can be obtained through both shell bending energy (8) and the Helfrich bending energy (9). We also note that the energy matching method is much less computationally expensive than direct atomistic simulations. Moreover, this method could be applied to problems where material parameters undergo drastic spatial variations. In this case, we can apply the energy method to inhomogeneous materials (e.g., assembly of different proteins) on subdomains where parameters are approximately constant.

The accurate elastic energy for the $\beta$-tapes not only allows us to explore higher order self-assembled structures - ribbons, fibrils, and fibers - but also opens a new avenue for more efficient investigations of dislocation and fracture for the self-assembled fibers and inelastic behavior such as slippage between $\beta$-tapes. These will be addressed in future work.

Acknowledgments. This work started while Leonid Berlyand was visiting the Max Planck Institute in Leipzig by invitation of Stefan Müller. He is grateful for the financial support and hospitality received during this visit. Lei Zhang and Maxim Fedorov acknowledge Giovanni Bellesia for the help on atomistic simulations. Part of this work was completed while Lei Zhang and Leonid Berlyand were visiting the Chinese Academy of Sciences. They thank their host, Pingbing Ming, and his institution for the kind invitation and hospitality.

\section{REFERENCES}

[1] A. Aggeli, M. Bell, N. Boden, J. N. Keen, P. F. Knowles, T. C. B. Mcleish, M. Pitkeathly, And S. E. Radford, Responsive gels formed by the spontaneous selfassembly of peptides into polymeric/beta-sheet tapes, Nature, 386 (1997), pp. 259-262.

[2] A. Aggeli, M. Bell, N. Boden, J. N. Keen, T. C. B. McLeish, I. Nyrkova, S. E. Radford, And A. Semenov, Engineering of peptide/beta-sheet nanotapes, J. Mater. Chem., 7 (1997), pp. $1135-1145$.

[3] A. Aggeli, M. Bell, L. M. Carrick, C. W. G. Fishwick, R. Harding, P. J. Mawer, S. E. Radford, A. E. Strong, And N. Boden, $p H$ as a trigger of peptide/beta-sheet selfassembly and reversible switching between nematic and isotropic phases, J. Amer. Chem. Soc., 125 (2003), pp. 9619-9628.

[4] A. Aggeli, I. A. Nyrkova, M. Bell, R. Harding, L. Carrick, T. C. B. Mcleish, A. N. SEMENOV, AND N. BODEN, Hierarchical self-assembly of chiral rod-like molecules as a model for peptide/beta-sheet tapes, ribbons, fibrils, and fibers, Proc. Natl. Acad. Sci. USA, 98 (2001), pp. 11857-11862.

[5] B. Alberts, A. Johnson, J. Lewis, M. Raff, K. Roberts, and P. Walter, Molecular Biology of the Cell, 4th ed., Garland Science, New York, 2002.

[6] G. Allaire, Shape Optimization by the Homogenization Method, Springer, Berlin, 2001.

[7] M. Arroyo and T. Belytschko, Finite element methods for the non-linear mechanics of crystalline sheets and nanotubes, Internat. J. Numer. Methods Engrg., 59 (2003), pp. 419456. 
[8] I. BABUŠKA AND S. A. SAUTER, Efficient solution of anisotropic lattice equations by the recovery method, SIAM J. Sci. Comput., 30 (2008), pp. 2386-2404.

[9] I. BABUŠKa AND S. A. SAUTER, Efficient solution of lattice equations by the recovery method part I: Scalar elliptic problems, Comput. Vis. Sci., 7 (2004), pp. 113-119.

[10] G. Bellesia, M. V. Fedorov, Y. A. Kuznetsov, and E. G. Timoshenko, Structure and stability of chiral beta-tapes: A computational coarse-grained approach, J. Chem. Phys., 122 (2005), 134901.

[11] G. Bellesia, M. V. Fedorov, And E. G. Timoshenko, Molecular dynamics study of structural properties of $\beta$-sheet assemblies formed by synthetic de novo oligopeptides, Phys. A., 373 (2007), pp. 455-476.

[12] G. Bellesia, M. V. Fedorov, and E. G. Timoshenko, Structural transitions in model betasheet tapes, J. Chem. Phys., 128 (2008), 195105.

[13] G. Bellesia and J. E. Shea, Self-assembly of beta-sheet forming peptides into chiral fibrillar aggregates, J. Chem. Phys., 126 (2007), 245104.

[14] G. Bellesia and J. E. Shea, Diversity of kinetic pathways in amyloid fibril formation, J. Chem. Phys., 131 (2009), 111102.

[15] A. Bensoussan, J. L. Lions, and G. Papanicolaou, Asymptotic Analysis for Periodic Structure. North-Holland, Amsterdam, 1978.

[16] L. Berlyand and H. Owhadi, A New Approach to Homogenization with Arbitrarily Rough Coefficients for Scalar and Vectorial Problems with Localized and Global Pre-computing, arXiv 0901.1463, 2009.

[17] X. Blanc, C. Le Bris, and P. L. Lions, Atomistic to continuum limits for computational materials science, M2AN Math. Model. Numer. Anal., 41 (2007), pp. 391-426.

[18] X. Blanc, C. Le Bris, And P.-L. Lions, From molecular models to continuum mechanics, Arch. Ration. Mech. Anal., 164 (2002), pp. 341-381.

[19] M. Born And K. Huang, Dynamical Theory of Crystal Lattices, Oxford University Press, Oxford, 1954.

[20] E. CANCÈS, C. Le BRIS, AND P.-L. Lions, Molecular simulation and related topics: Some open mathematical problems, Nonlinearity, 21 (2008), pp. T165-T176.

[21] D. Coeurjolly, S. Miguet, and L. Tougne, Discrete curvature based on osculating circle estimation, in Proceedings of Visual Form 2001, Lecture Notes in Comput. Sci. 2059, Springer, Berlin, 2001.

[22] S. Conti, G. Dolzmann, B. Kirchheim, And S. Müller, Sufficient conditions for the validity of the Cauchy-Born rule close to so(n), J. Eur. Math. Soc. (JEMS), 8 (2006), pp. 515-539.

[23] P. Donato and D. Cioraescu, An Introduction to Homogenization, Oxford University Press, Oxford, 1999.

[24] W. E, B. Engquist, X. Li, W. Ren, And E. VAnden-Eijnden, The heterogeneous multiscale method: A review, Comm. Comput. Phys., 2 (2007), pp. 367-450.

[25] W. E And P. Ming, Cauchy-Born rule and the stability of crystalline solids: Static problems, Arch. Ration. Mech. Anal., 183 (2007), pp. 241-297.

[26] C. W. G. Fishwick, A. J. Beevers, L. M. Carrick, C. D. Whitehouse, A. Aggeli, and N. Boden, Structures of helical beta-tapes and twisted ribbons: The role of side-chain interactions on twist and bend behavior, Nano Lett., 3 (2003), pp. 1475-1479.

[27] G. Friesecke And R. D. James, A scheme for the passage from atomic to continuum theory for thin films, nanotubes and nanorods, J. Mech. Phys. Solids, 48 (2000), pp. 15191540.

[28] G. Friesecke And F. Theil, Validity and failure of the Cauchy-Born hypothesis in a twodimensional mass-spring lattice, J. Nonlinear Sci., 12 (2002), pp. 445-478.

[29] N. M. Ghoniem, E. P. Busso, N. Kioussis, And H. C. Huang, Multiscale modelling of nanomechanics and micromechanics: An overview, Philos. Mag., 83 (2003), pp. 3475-3528.

[30] E. Grinspun, A. N. Hirani, M. Desbrun, And P. Schröder, Discrete shells, in Proceedings of the 2003 ACM SIGGRAPH/Eurographics Symposium on Computer Animation, 2003, pp. $62-67$.

[31] W. Helfrich, Elastic properties of lipid bilayers-theory and possible experiment, Z. Naturforsch. C, 28 (1973), pp. 693-703.

[32] K. Imata, T. Fujiwara, Y. Matsuki, H. Akutsu, S. Takahashi, H. Naiki, and Y. Goto, $3 d$ structure of amyloid protofilaments of beta(2)-microglobulin fragment probed by solid-state nmr, Proc. Natl. Acad. Sci. USA, 103 (2006), pp. 18119-18124.

[33] V. V. Jikov, S. M. Kozlov, And O. A. Oleinik, Homogenization of Differential Operators and Integral Functionals, Springer, Berlin, 1991.

[34] L. Kharevych, P. Mullen, H. Owhadi, and M. Desbrun, Numerical coarsening of inhomogeneous elastic materials, ACM Trans. Graphics, 28 (2009), Article 51.

Copyright $@$ ( ) by SIAM. Unauthorized reproduction of this article is prohibited. 
[35] T. Langer, A. G. Belyaev, And H.-P. Seidel, Asymptotic analysis of discrete normals and curvatures of polylines, in Proceedings of the 21st Spring Conference on Computer Graphics, ACM, New York, 2005, pp. 229-232.

[36] Q. Lu, M. Arroyo, And R. Huang, Elastic bending modulus of monolayer graphene, J. Phys. D: Appl. Phys., 42 (2009), 102002.

[37] D. M. Marini, W. Hwang, D. A. Lauffenburger, S. G. Zhang, and R. D. Kamm, Lefthanded helical ribbon intermediates in the self-assembly of a $\beta$-sheet peptide, Nano Lett., 2 (2002), pp. 295-299.

[38] M. Meyer, M. Desbrun, P. Schröder, And A. H. Barr, Discrete differential-geometry operators for triangulated 2-manifolds, in Visualization and Mathematics III, H.-C. Hege and K. Polthiers, eds., Springer, Heidelberg, 2003, pp. 35-57.

[39] F. Muller-Plathe, Coarse-graining in polymer simulation: From the atomistic to the mesoscopic scale and back, ChemPhysChem, 3 (2002), pp. 754-769.

[40] J. Nocedal and S. J. Wright, Numerical Optimization, Springer, Berlin, 1999.

[41] H. Owhadi And L. Zhang, Metric based upscaling, Comm. Pure Appl. Math., 60 (2007), pp. 675-723.

[42] G. Panasenko and N. Bakhvalov, Homogenisation: Averaging Processes in Periodic Media, Math. Appl. 36, Kluwer Academic Publishers, Dordrecht, the Netherlands, 1990.

[43] J. C. Phillips, R. Braun, W. Wang, J. Gumbart, E. Tajkhorshid, E. Villa, C. Chipot, R. D. Skeel, L. KAle, And K. Schulten, Scalable molecular dynamics with NAMD, J. Comput. Chem., 26 (2005), pp. 1781-1802.

[44] B. Sснмidt, Effective Theories for Thin Elastic Films, Ph.D. thesis, University of Leipzig, 2006.

[45] L. C. Serpell, M. Sunde, and C. C. F. Blake, The molecular basis of amyloidosis, Cell. Mol. Life Sci., 53 (1997), pp. 871-887.

[46] S. Shu, I. BabušKa, Y. XiaO, J. Xu, and L. Zikatanov, Multilevel preconditioning methods for discrete models of lattice block materials, SIAM J. Sci. Comput., 31 (2008), pp. 687-707.

[47] J. M. Sullivan, Curvatures of smooth and discrete surfaces, in Oberwolfach Seminars, Discrete Differ. Geom. 38, Birkhäuser, Basel, Switzerland, 2008, pp. 175-188.

[48] D. Terzopoulos, J. Platt, A. Barr, and K. Fleischer, Elastically deformable models, Comput. Graphics, 21 (1987), pp. 205-214.

[49] X. Q. WANG AND Q. Du, Modelling and simulations of multi-component lipid membranes and open membranes via diffuse interface approaches, J. Math. Biol., 56 (2008), pp. 347-371.

[50] Z. Yang AND W. E, Generalized Cauchy-Born rules for elastic deformation of sheets plates, and rods: Derivation of continuum models from atomistic models, Phys. Rev. B, 74 (2006), 184110.

[51] S. G. ZHANG, Emerging biological materials through molecular self-assembly, Biotechnol. Adv., 20 (2002), pp. 321-339.

[52] S. G. Zhang, D. M. Marini, W. Hwang, and S. SAntoso, Design of nanostructured biological materials through self-assembly of peptides and proteins, Curr. Opin. Chem. Biol., 6 (2002), pp. 865-871.

[53] A. ZoRin, Curvature-based energy for simulation and variational modeling, in Proceedings of the International Conference on Shape Modeling and Applications 2005, IEEE Computer Society, Los Alamitos, CA, 2005.

Copyright (c) by SIAM. Unauthorized reproduction of this article is prohibited. 